\title{
THE POTENTIAL J-RELATION AND AMALGAMATION BASES FOR FINITE SEMIGROUPS
}

\author{
T. E. HALL AND MOHAN S. PUTCHA
}

\begin{abstract}
Let $S$ be a finite semigroup, $a, b \in S$. When does there exist a finite semigroup $T$ containing $S$ such that $a \mathrm{~J} b$ in $T$ ? This problem was posed to the second named author by John Rhodes in 1974. We show here that if $a$, $b$ are regular, then such a semigroup $T$ exists if and only if either $a J b$ in $S$, or $a \notin S b S$ and $b \notin S a S$. We use this result to show that analgamation bases for the class of finite semigroups have linearly ordered $J$-classes.
\end{abstract}

1. Preliminaries. For $X$ any set, $|X|$ denotes the cardinality of $X$ and $\tau(X)$ denotes the full transformation semigroup on $X$, acting on the right on $X$. If $a \in T(X)$, then $\rho(a)$ denotes the rank of $a$, namely $|X a|$. Let $F$ be a field. Then we let $M_{n}(F)$ denote the multiplicative monoid of all $n \times n$ matrices over $F$. If $a \in M_{n}(F)$, then $\rho(a)$ denotes the rank of $a$. Let $X$ be a set, with $|X|=n$. Then $\tau(X)$ acts naturally on a vector space of dimension $n$ over $F$. Thus $T(X)$ embeds naturally into $M_{n}(F)$. Moreover, this embedding preserves rank.

Let $S$ be a semigroup. If $a, b \in S$, then we write $a \mid b$ (a divides $b$ ) if $b \in S^{1} a S^{1}$, i.e., if $J_{b} \leq J_{a}$.

By an amalgam we mean a list $(S, T ; U)$ of semigroups such that $S \cap T=U$, and we say the amalgam is embeddable if there is a semigroup $W$ with $S$ and $T$ as subsemigroups (see [5] for more details). By an amalgamation base for a class $C$ of semigroups we mean any $U \in C$ such that every amalgam $(S, T ; U)$ with $S, T \in \mathcal{C}$ is embeddable in some $W \in C$ (see [4] for some examples).

\section{The potential $J$-relation.}

THEOREM 1. Let $S$ be a finite semigroup, $A \subseteq S$.

(a) If for any $a, b \in A$, either $a J b$ or $a \nmid b, b \nmid a$, then there exists a finite semigroup $T$ containing $S$ such that $A$ lies in a J-class of $T$. If $S$ is an inverse semigroup, then $T$ can be chosen to be an inverse semigroup.

(b) Conversely, if $S$ can be embedded in a finite semigroup $T$ containing $A$ within $a \mathrm{~J}$-class and if every element of $A$ is regular in $S$, then for any $a, b \in A$, either $a J b$ in $S$ or $a \nmid b, b \nmid a$ in $S$.

Proof. (a) Without loss of generality we can assume that $S=S^{1}$ and that $A$ contains no pair of $J$-equivalent elements. We shall embed $S$ in a transformation semigroup $\tau(X)$ such that the elements of $A$ all have the same rank, i.e., are $J$-related.

Choose $a \in A$ such that $|S a|$ is the maximum. Let $A=\left\{a=a_{0}, a_{1}, \ldots, a_{p}\right\}$. Let $I_{j}=\bigcup_{k \neq j} S a_{k} S, j=1, \ldots, p$. Let $S_{0}=S, S_{j}=S / I_{j}, j=1, \ldots, p$. Then $S$ acts on the right on $S_{i}, i=0, \ldots, p$. Let $\left|S a_{i}\right|=m_{i}, i=0, \ldots, p, \beta_{j}=\left|S_{j} a_{j}\right|>1$,

Received by the editors December 12, 1984.

1980 Mathematics Subject Classification. Primary 20M10.

(C) 1985 American Mathematical Society $0002-9939 / 85 \$ 1.00+\$ .25$ per page 
$j=1, \ldots, p, \alpha_{0}=\prod_{j=1}^{p}\left(\beta_{j}-1\right)$ and $\alpha_{j}=\alpha_{0}\left(m_{0}-m_{j}\right) /\left(\beta_{j}-1\right), j=1, \ldots, p$. So $\alpha_{0} \geq 1, \alpha_{j} \geq 0, j=1, \ldots, p$. Let $X$ denote the disjoint union of $\alpha_{i}$ copies of $S_{i}, i=0, \ldots, p$. Then $S$ acts faithfully on the right on $X$ and thus $S$ embeds in $\tau(X)$. Clearly $\left|X a_{0}\right|=\alpha_{0} m_{0}+\sum_{j=1}^{p} \alpha_{j}$, and $\left|X a_{j}\right|=\alpha_{0} m_{j}+\alpha_{j} \beta_{j}+\sum_{k \neq 0, j} \alpha_{k}$, $j=1, \ldots, p$. Routine calculations now show that $\left|X a_{0}\right|=\left|X a_{j}\right|, j=1, \ldots, p$. So $A$ lies within a $J$-class of $T(X)$.

If $S$ is an inverse semigroup, then we can use the Preston-Vagner representation of $S$ and the $S_{j}$ 's to obtain a representation of $S$ in the symmetric inverse semigroup on $X$.

(b) Suppose that $S$ can be so embedded in $T$ and suppose, to the contrary, that there exist $a, b \in A$ such that $a \mid b$ and $b \nmid a$. By [2, Theorem 1 or 7, Proposition 3.1] there exist idempotents $e \in J_{a}, f \in J_{b}$ such that $e>f$. Clearly $e J f$ in $T$, so $T$ contains a copy of the bicyclic semigroup [1, Theorem 2.54] and hence is infinite, a contradiction.

The next theorem shows that with respect to semigroup division, the whole semigroup can be put into a $J$-class.

THEOREM 2. Let $S$ be a finite semigroup. Then there exists a finite regular semigroup $T$, a subsemigroup $T_{0}$ of $T$, a J-class $J$ of $T$ and a morphism $\phi: T_{0} \rightarrow S$ such that $\phi\left(J \cap T_{0}\right)=S$. If $S$ is a regular semigroup, then $T_{0}$ can be chosen to be a regular semigroup. If $S$ is an inverse semigroup, then $T_{0}$ and $T$ can be chosen to be inverse semigroups.

ProOF. Let $T_{0}=S \times\{0,1\}$ with the following multiplication:

$$
(a, \alpha)(b, \beta)=(a b, \gamma)
$$

where

$$
\gamma= \begin{cases}1 & \text { if } \alpha=\beta=1 \text { and } a J b J a b, \\ 0 & \text { otherwise. }\end{cases}
$$

It can be verified that $T_{0}$ is a semigroup. If $S$ is a regular semigroup, then $T_{0}$ is a regular semigroup. If $S$ is an inverse semigroup, then $T_{0}$ is an inverse semigroup. The map $a \mapsto(a, 0)$ embeds $S$ into $T_{0}$.

Easy manipulation (or Remark 1 below) now shows that the subset $A=S \times\{1\}$ satisfies the hypothesis of Theorem 1(a). Thus there exists a finite semigroup $T$ containing $T_{0}$ such that $A$ lies in a $J$-class $J$ of $T$. the map $\phi: T_{0} \rightarrow S$ given by $\phi(a, \alpha)=a$ is a morphism and $\phi\left(J \cap T_{0}\right) \supseteq \phi(A)=S$. This proves the theorem.

REMARK 1. In fact $S \times\{0\}$ is an ideal of $T_{0}$ and $T_{0} /(S \times\{0\})$ is isomorphic to the 0 -direct union $[\mathbf{1}, \S 6.3]$ of all the principal factors $\{J \cup\{0\}: J \in S / J\}$ (including, if $S=S^{0}$, the two element semilattice), while $T_{0}$ is the ideal extension of $S \times\{0\}$ (or $S)$ by $T_{0} /(S \times\{0\})$ determined by the partial morphism $(s, 1) \mapsto(s, 0)$ for $s \in S$.

\section{Amalgamation bases.}

THEOREM 3. For any amalgamation base of the class of finite [finite regular, finite inverse] semigroups, the J-classes are linearly ordered.

Proof. Take any amalgamation base, $U$ say, of the class of finite semigroups, and suppose, to the contrary, that there are two elements, $a$ and $b$ say, whose $J$-classes are not comparable.

Case I. $a$ or $b$ is regular, say $a$. Take any idempotent $e \in J_{a}$. Form a semigroup $U^{\prime}=U \cup\left\{e^{\prime}\right\}$ containing $U$ as a subsemigroup (where $e^{\prime} \notin U$ ) by defining $e^{2}=$ 
$e^{\prime}, e^{\prime} u=e u, u e^{\prime}=u e$, for all $u \in U$. Then $e^{\prime} \nmid b$ and $b \nmid e^{\prime}$ in $U^{\prime}$, so by Theorem 1 there exists a finite semigroup $S$ containing $U^{\prime}$ such that $c^{\prime} J b$ in $S$.

Since $J_{e}=J_{a}$ and $J_{b}$ are not comparable, again by Theorem 1 , there exists a finite semigroup $T$ containing $U$ such that $e J b$ in $T$.

The amalgam $(S, T ; U)$ is embeddable in a finite semigroup, $W$ say, since $U$ is an amalgamation base for the class of finite semigroups. But then in $W$ we have $e J b J e^{\prime}$ and $e<e^{\prime}$, whence $W$ contains a copy of the bicyclic semigroup [1, Theorem 2.54] and is infinite, a contradiction.

The proof so far is easily modified to give a proof of the bracketed statements. Note that the result for the class of finite regular semigroups is a trivial corollary of the result for the class of finite semigroups, since a regular semigroup is an amalgamation base for either class if and only if it is one for the other class (since any finite semigroup embeds in a finite regular semigroup).

$C a s e$ II. $a$ and $b$ are not regular. Without loss of generality we can assume that $U$ is a subsemigroup of $M_{n}\left(\mathbf{Z}_{2}\right)$ for some positive integer $n$, and that $n>\rho(a) \geq$ $\rho(b)$. Consider the embedding $\theta: M_{n}\left(\mathbf{Z}_{2}\right) \rightarrow M_{2 n}\left(\mathbf{Z}_{2}\right)$ given by $\theta(c)=\left(\begin{array}{ll}c & 0 \\ 0 & c\end{array}\right)$. Put $a^{\prime}=\left(\begin{array}{ll}0 & 1 \\ a & 0\end{array}\right)$, where 1 denotes the $n \times n$ identity matrix; then $\rho\left(a^{\prime}\right)=n+\rho(a)>$ $2 \rho(a)=\rho(\theta(a)) \geq \rho(\theta(b))$ and $a^{\prime 2}=\theta(a)$. (This method of finding square roots is a variation of that due to C. J. Ash [5, Theorem 5.1].) Thus, so far, we have embedded $U$ in a finite semigroup $U^{\prime}$ with an element $a^{\prime}$ such that $a^{\prime 2}=a$ and $b \nmid a^{\prime}$ (note that from $b \nmid a$ in $U$ we do not get $b \nmid a$ in $U^{\prime}$, so it is not immediate from $a^{\prime 2}=a$ that $b \nmid a^{\prime}$ in $\left.U^{\prime}\right)$.

Now consider the embedding $\psi: U \rightarrow U^{\prime} \times\left(U / U^{1} a U^{1}\right)$ given by $\psi(u)=(u, \phi(u))$, where $\phi$ is the canonical morphism of $U$ upon $U / U^{1} a U^{1}$. Put $v=\left(a^{\prime}, 0\right)$; then $v^{2}=(a, 0)=\psi(a)$ and $v \nmid(b, \phi(b))=\psi(b)$ since $\phi(b) \neq 0$. Also $\psi(b) \nmid v$ since $b \nmid a^{\prime}$ in $U^{\prime}$. Thus we have a semigroup $V$ containing $U$ and an element $v$ such that $v^{2}=a, v \nmid b, b \nmid v$.

By Theorem 1, there exists a finite semigroup $S$ containing $V$ such that $v \mathrm{~J} b$ in $S$. Also, since in $U, J_{a}$ and $J_{b}$ are not comparable, by Theorem 1 there is a finite semigroup $T$ containing $U$ such that $a J b$ in $T$.

Since $U$ is an amalgamation base for the class of finite semigroups, the amalgam $(S, T ; U)$ is embeddable in a finite semigroup $W$, say. Then $v J b J a=v^{2}$ in $W$, and since $W$ is finite, we have $v \nLeftarrow v^{2}=a$ in $W$. Thus $a$ is in a subgroup of $W$ and hence in a subgroup of $U$, contradicting that $a$ is not regular in $U$.

REMARK 2. The existence of a finite inverse semigroup which is not an amalgamation base for the class of finite inverse semigroups was first shown by C. J. Ash: his example, given in [3], is the three element semilattice which is not a chain. His construction and proof led us to the proof in Case I above.

REMARK 3. One of the authors has recently shown that the J-classes being linearly ordered is also a sufficient condition for a finite inverse semigroup to be an amalgamation base of the class of finite inverse semigroups.

\section{REFERENCES}

1. A. H. Clifford and G. B. Preston, The algebraic theory of semigroups, Math. Surveys, I and II, Amer. Math. Soc., Providence, R. I., 1961 and 1967.

2. T. E. Hall, On the natural ordering of J-classes and of idempotents in a regular semigroup, Glasgow Math. J. 11 (1970), 167-168. 
3. __ Free products with amalgamation of inverse semigroups, J. Algebra 34 (1975), 375-385.

4. __ Representation extension and amalgamation for semigroups, Quart. J. Math. Oxford Ser. (2) 29 (1978), 309-334.

5. _ Inverse and regular semigroups and amalgamation: a brief survey, Symposium on Regular Semigroups, De Kalb, Illinois, 1979, pp. 49-79.

6. J. M. Howie, An introduction to semigroup theory, London Math. Soc. Monographs 7, Academic Press, London, 1976.

7. J. Rhodes, Some results on finite semigroups, J. Algebra 4 (1966), 471-504.

Department of Mathematics, Monash University, Clayton, Victoria 3168, Australia

Department of Mathematics, North Carolina State UNiversity, Raleigh, NORTH CAROLINA 27695 